\title{
Kimura Disease of the Parotid Gland: Management Approach
}

\author{
Hafeza MS, Vanitha Palanisamy \\ Department of Otorhinolaryngology, Department of Otorhinolaryngology, Hospital Sultanah Aminah, Johor Bahru, Malaysia.
}

*Corresponding Author: Hafeza MS, Department of Otorhinolaryngology, Department of Otorhinolaryngology, Hospital Sultanah Aminah, Johor Bahru, Malaysia.

\section{Received date: August 08, 2021: Accepted date: December 12, 2021: Published date: January 03,2022}

Citation: Hafeza MS, Vanitha Palanisamy (2022) Kimura Disease of the Parotid Gland: Management Approach, Journal of Clinical Otorhinolaryngology 4(1); DOI: 10.31579/2692-9562/038

Copyright: () 2022, Hafeza MS. This is an open access article distributed under the Creative Commons Attribution License, which permits unrestricted use, distribution, and reproduction in any medium, provided the original work is properly cited.

\begin{abstract}
Here we present a 43-years old Malay man with Kimura's disease of the right parotid gland. Asian men have a predisposition for this chronic inflammatory disorder of unknown etiology mostly presenting at the head and neck region. The management in dealing with this patient is not straight forward as the presentation mimics a subcutaneous tumor-like mass in which main differential diagnosis includes lymphoproliferative disorders and parotid neoplasm. Moreover, cytological investigation is inadequate, and this necessitates the use intra-operative frozen section prior to tumor debulking. Without proper investigation, misdiagnosis can occur and potentially expose the patient to unnecessary extensive surgical procedures. Here we discuss step by step approach in managing this patient.
\end{abstract}

Keywords: parotid; kimura; swelling; benign

\section{Introduction}

Kimura's disease is a rare, benign disorder associated with chronic inflammatory lesions of unclear etiology [1]. It classically presents as painless subcutaneous nodule in the head and neck region associated with regional lymphadenopathy and/or salivary gland involvement, peripheral hypereosinophilia and elevated serum IgE level [1]. It is predominantly seen in Asian males during their second and fourth decades of life [1]. Due to its rarity, most of the clinical studies are based on clinical reports and case series in Asian literature. Early diagnosis of Kimura's disease could prevent unnecessary and potentially harmful diagnostic procedures. However, diagnosis can be difficult due to its histological similarity to benign or malignant diseases. Herein, we report a rare case of Kimura disease with parotid gland involvement, and we will discuss the approach in reaching the diagnosis and the management after.

\section{Case Presentation}

A 43-year-old Malay male with no known medical illness first presented to use with painless swellings at his right forehead and right pre-auricular region. These swellings were gradually increasing in size for the past 3 years. Patient had no family history of malignancy and denies constitutional symptoms.

On examination, the right forehead swelling measured $4.0 \times 4.0 \mathrm{~cm}$ whereas the right pre-auricular swelling measured $6.0 \times 5.0 \mathrm{~cm}$. Both the swellings were non-tender and firm in consistency. Hyperpigmentation was observed only over the right forehead lesion. Otherwise, no axillary or inguinal lymphadenopathy noted. Facial nerve is intact and other systemic examinations were normal.

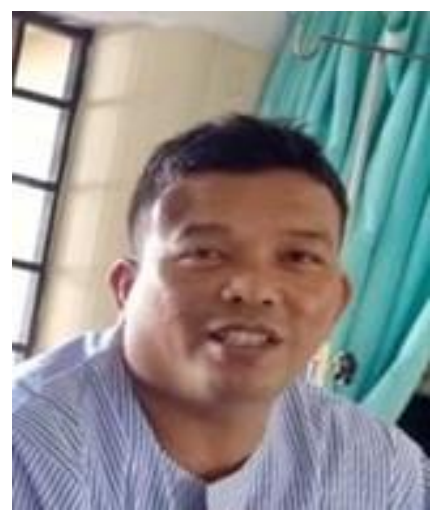

Figure 1

Blood investigation including full blood count, renal profile, and serum immunoglobulin E (IgE) level were within normal range. Chest radiography had no significant findings. Fine-needle aspiration cytology (FNAC) showed polymorphous lymphoid population with scattered neutrophils and eosinophils. There were no Reed-Sternberg cells and no deposition of proteinaceous material seen. Features were suggestive of a lymphoproliferative lesion, but we were unable to ascertain if it is reactive or neoplastic in nature.

We proceeded with a CT neck which showed multiple enhancing lesions within the superficial and deep lobe of right parotid gland. The mass had a clear plane with right masseter muscle and had no overlying bony or skin involvement. Multiple sub-centimeter intra-parotid lymph 
nodes were also identified at right and left parotid gland. The right forehead lesion showed no adjacent bony erosion or sclerosis. Bilateral submandibular glands were normal.

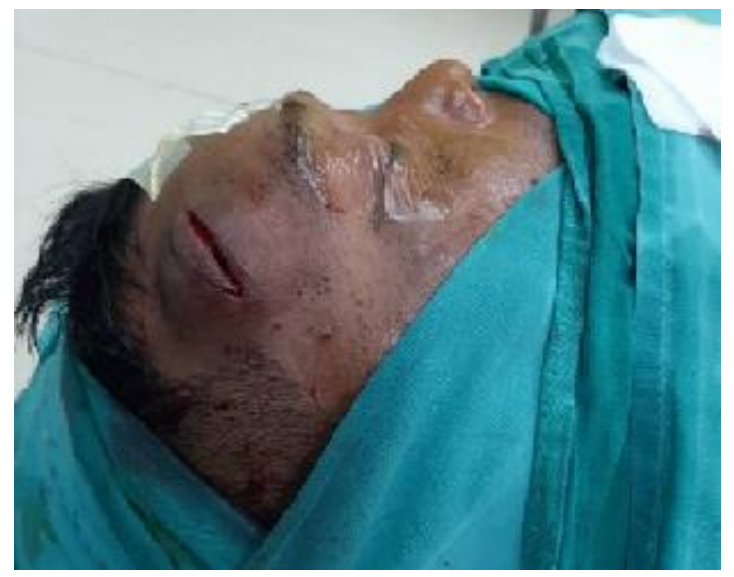

Figure 2

With the initial investigations above, there is a high index suspicion for lymphoma or parotid neoplasm. A wedge biopsy was performed and sent for intra-operative frozen section. Differential diagnosis based on the frozen section were in favor for 1) lymphoproliferative disorder and 2) squamous cell carcinoma. Tumor debulking was then performed over the right parotid mass. Intraoperatively, the firm fibrotic mass infiltrates subcutaneous tissue and both superficial and deep gland with presence of intra-parotid nodes. Facial nerve identified and preserved during surgery. Excision of the forehead lesion was performed under the same setting.

Histopathological report finally confirmed the diagnosis of Kimura's disease of the right parotid gland and right forehead lesion. Intra-parotid lymph node identified within the wedge biopsy done showed follicular hyperplasia with prominent germinal centers and abundant eosinophilic infiltration. Occasional Warthin-Finkeldey cells were observed. Salivary glandular tissue was unremarkable.

\section{Outcome and Follow-Up}

Patient remains disease free 2 years post-surgery. He developed a new subcutaneous lesion overlying the right trapezius muscle measuring about $1.0 \times 1.0 \mathrm{~cm}$. A trial of low dose prednisolone was given to the patient with no enlargement or progression of the lesion. He is still under our regular follow-up.

\section{Discussion}

Kimura's disease was first described in 1937 by Kimm and Szeto in Chinese literature as "eosinophilic hyperplastic lymphogranuloma". Subsequently, Kimura et al. described the pathological features of this disease in 1948, and it is widely recognized as Kimura's disease [1]. The incidence peaks during the second and fourth decades of life and majority of patients were Asian men [1]. Two retrospective case series done in China has shown the ratio of male to female was 5:1 [2,3].
Most cases are unilateral but bilateral cases have been reported with a ratio of $4: 1[2,3]$.

The common mode of clinical presentation is in the form of painless subcutaneous nodules usually found in the head and neck region. These nodules may be associated with pruritus, dermatitis, and hyperpigmentation. Head and neck region is most affected in Kimura disease as there are numerous lymph nodes present. Subcutaneous nodules in the head and neck region accounts for about $70 \%$ whereas the extremities about $12 \%$ and trunk only $3 \%$ [10]. This disease is most frequently associated with regional lymphadenopathy and/or salivary gland involvement.

Kimura disease is an immune-mediated inflammatory disorder of unknown etiology. It. It is believed that the clinical presentations may be contributed by inflammation, endocrinal disorders, autoimmune diseases, parasite infestation, viral infections, and allergies [3]. The aberrant immune reaction that is believed to be the root of Kimura's disease also predisposes the patient to allergic conditions like asthma, chronic urticaria, pruritus and rhinitis as well as presence of peripheral hypereosinophilia and markedly elevated serum IgE levels [8]. Pruritus or melanin pigmentation of the overlying skin is probably due to nerve infiltration by lymphocytes and eosinophils [4]. Primarily, it is a localized disorder, but sometimes may be associated with systemic manifestations. Renal involvement is the commonest systemic manifestation. Up to $60 \%$ of these patients exhibit renal involvement manifesting as extra membranous glomerulonephritis and nephrotic syndrome.

Clinically, Kimura's disease lesions mimic neoplasms in the head and neck region and misdiagnosis are common. Thus, it necessitates an extensive investigation before accurate diagnosis can be made. CT and MRI can be useful for diagnosing Kimura's disease in the head and neck areas however they are not specific [5]. The imaging differential diagnosis for Kimura's disease in the head and neck includes parotid tumors, lymphoma, metastatic tumors, malignant tumors of cutaneous or subcutaneous origin, tuberculosis, and ALHE [5]. Characteristic imaging results include multiple ill-defined enhanced masses within and around the parotid gland with associated regional lymphadenopathy. However, parotid tumors are usually encapsulated or pseudoencapsulated and are limited to the parotid gland, while Kimura's disease is frequently associated with irregular extension into the subcutaneous tissue. Since CT findings in Kimura disease and lymphoma can be similar, a biopsy of the lymph node is essential.

FNAC is useful as an initial investigation of Kimura's disease, but histopathological examination is required for definitive diagnosis as cytology may sometimes be difficult to interpret. In our study, FNAC failed to yield a definite diagnosis. Decision for wedge biopsy and tumor debulking of the parotid gland was based on the intra-operative frozen section result.

Pathological examination is the golden standard for the diagnosis of Kimura's disease. The histopathologic aspect of Kimura's disease is marked by a triple component: cellular, fibrocartilaginous, and vascular [4]. Hui et al. classified the histological features of Kimura's disease as constant, frequent, and rare $[4,9]$. 


\begin{tabular}{|c|c|c|}
\hline Constant & Frequent & Rare \\
\hline 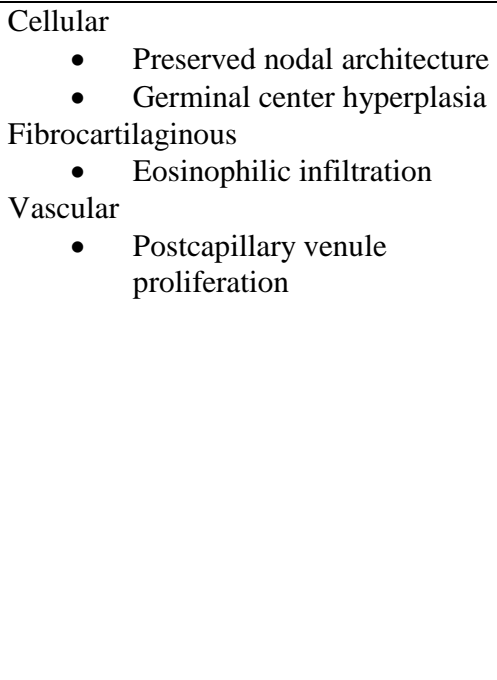 & $\begin{aligned} \text { Cellular } & \\
\text { - } & \text { Sclerosis } \\
\text { - } & \text { Polykaryocytes } \\
\text { - } & \text { Germinal center } \\
& \text { vascularization/necrosis } \\
\text { - } & \begin{array}{l}\text { Protein/IgE deposits in } \\
\text { germinal centers }\end{array} \\
\text { Fibrocartilaginous } & \text { Eosinophilic microabscess } \\
\text { - } & \text { Capsular fibrosis with } \\
\text { Vascular } & \text { subcapsular sinusoid } \\
\text { - } & \begin{array}{l}\text { Perinoditeration } \\
\text { involvement }\end{array} \\
& \end{aligned}$ & $\begin{array}{l}\text { Cellular } \\
\text { - } \quad \text { Progressive transformation of } \\
\text { germinal centers }\end{array}$ \\
\hline
\end{tabular}

Surgical excisions have been considered the golden standard of treatment for Kimura's disease. The role of surgery is mainly for diagnostic and cosmetic purposes. As Kimura's disease is benign, surgical resection does not spread lesions. Other treatment options include surgical resection, cytotoxic and radiation therapy as well as regional and or systemic steroids therapy. However, there is no consensus or standardized treatment protocol for Kimura's disease as recurrence rate is high. In a study of 21 patients with Kimura's disease, the overall recurrence rate was $46.8 \%$ [3]. The neck and parotid gland were the most likely to relapse [3]. A combination of surgery with postoperative intervention seems to be a reasonable approach for treatment of Kimura's disease. This has been proven by Ye at al. and Chang et al. with local recurrence rate of $8.1 \%$ and $35.7 \%$ in patients who underwent surgical excision followed by low-dose radiotherapy $[6,11]$.

For our patient in this case, he developed a small subcutaneous nodule over his back overlying the right trapezius muscle 2 months postsurgery. He was started on low dose prednisolone 30mg OD for 2 weeks and the nodule has not increased in size. There is no evidence of relapse 2 years post-surgery. Steroid therapy is carried out as a secondline treatment in consideration of the risks of relapse and side-effects. Nakahara et al. reported that steroid therapy can control lesions, lymphadenopathy, and nephrotic syndrome in Kimura's disease, but local recurrence occurred frequently during the period of steroid dose tapering [7].

\section{Conclusion}

Kimura's disease although rare in entity should always be included as a differential for head and neck masses and lymphadenopathy. It follows an indolent course and misdiagnosis is common. Histopathological examination is the gold standard for diagnosis of Kimura's disease. Although there is no consensus regarding optimal treatment and recurrence rate is high, prognosis is good with no reports on malignant transformation.

\section{References}

1. Ye P, Ma DQ, Yu GY, Gao Y, Peng X. Comparison of the efficacy of different treatment modalities for Kimura's disease. Int J Oral Maxillofac Surg. 2017;46(3):350-4.

2. Li TJ, Chen XM, Wang SZ, Fan MW, Semba I, Kitano M. Kimura's disease: a clinicopathologic study of 54 Chinese patients. Oral Surg Oral Med Oral Pathol Oral Radiol Endod. 1996;82(5):549-55.

3. Zhang, G., Li, X., Sun, G., Cao, Y., Gao, N., \& Qi, W. (2020). Clinical analysis of Kimura's disease in 24 cases from China. BMC Surgery, 20(1), NA.

4. Chen H, Thompson LD, Aguilera NS, Abbondanzo SL. Kimura disease: a clinicopathologic study of 21 cases. Am J Surg Pathol. 2004;28(4):505-13.

5. Park SW, Kim HJ, Sung KJ, Lee JH, Park IS. Kimura disease: $\mathrm{CT}$ and MR imaging findings. AJNR Am J Neuroradiol. 2012;33(4):784-8.

6. Ye P, Wei T, Yu GY, Wu LL, Peng X. Comparison of local recurrence rate of three treatment modalities for Kimura disease. J Craniofac Surg. 2016;27(1):170-4.

7. Nakahara C, Wada T, Kusakari J, Kanemoto K, Kinugasa H, Sibasaki M, et al. Steroid-sensitive nephrotic syndrome associated with Kimura disease. Pediatr Nephrol (Berlin, Germany). 2000;14(6):482-5.

8. Meningaud JP, Pitak-Arnnop P, Fouret P, Bertrand JC. Kimura's disease of the parotid region: report of 2 cases and review of the literature. J Oral Maxillofac Surg. 2007;65:134140.

9. Hui PK, Chan JK, Ng CS, Kung IT, Gwi E. Lymphadenopathy of Kimura's disease. Am J Surg Pathol. 1989;13:177-186

10. Terakado N, Sasaki A, Takebayashi T, et al. A case of Kimura's disease of the hard palate. Int $J$ Oral Maxillofac Surg. 2002;31:222- 224 .

11. Chang AR, Kim K, Kim HJ, Kim IH, Park CI, Jun YK. Outcomes of Kimura's disease after radiotherapy or nonradiotherapeutic treatment modalities. Int J Radiat Oncol Biol Phys 2006;65:1233-9. 